\title{
From Plaque to Pretzel: Fold Formation and Acetylcholine Receptor Loss at the Developing Neuromuscular Junction
}

\author{
Maria Julia Marques, José-Angel Conchello, and Jeff W. Lichtman \\ Department of Anatomy and Neurobiology, Washington University School of Medicine, St. Louis, Missouri 63110
}

Although there has been progress in understanding the initial steps in the formation of synapses, less is known about their subsequent maturation (Sanes and Lichtman, 1999). Two alterations on the postsynaptic side of the mammalian neuromuscular junction occur during early postnatal life: acetylcholine receptors (AChRs) disappear from parts of the developing junction as all but one axonal inputs are removed, and the topography of the postsynaptic membrane becomes more complicated as gutters and folds are established. We have studied the maturation of the AChR distribution and postsynaptic topography simultaneously by imaging labeled AChRs at the mouse neuromuscular junction in a new way, using reflected light confocal microscopy. At birth postsynaptic receptors were localized in irregular patches within a spoon-shaped plaque. Beginning several days later, receptor regions within a single endplate were divided into differentiated and less organized compartments. Folds generally oriented orthogonal to the long

The use of fluorescently tagged $\alpha$-bungarotoxin to label acetylcholine receptors (AChRs) at the neuromuscular junction has shown that there is a dramatic maturational alteration in receptor distribution in early postnatal life. At birth, AChRs are arranged in an oval plaque in what appears to be a relatively uniform density. Over several weeks this plaque perforates (Nyström, 1968; Steinbach, 1981; Slater, 1982; Balice-Gordon and Lichtman, 1993) such that the regions that maintain AChRs become the pretzel-shaped branches of the mature singly innervated neuromuscular junction, whereas regions that lose membraneassociated AChRs also lose nerve terminal innervation during the process of synapse elimination (Balice-Gordon and Lichtman, 1993; Gan and Lichtman, 1998). Although fluorescence labeling of AChRs allows for the study of the overall distribution of receptors during development, this technique has not been useful in studying alterations in the three-dimensional topography of the muscle fiber membrane as neuromuscular junctions mature. Hence, the formation of gutters (primary synaptic clefts) and the

Received Nov. 3, 1999; revised Feb. 18, 2000; accepted Feb. 28, 2000.

Financial support was received from Fundo de Amparo $\times$ Pesquisa do Estado de Sao Paulo (93/3419-7; 95/6110-2), Fundo de Apoio ao Ensino e à Pesquisa, and Conselho Nacional de Pesquisas (to M.J.M.) and the Muscular Dystrophy Association, National Institutes of Health, and Bakewell NeuroImaging Fund.

Correspondence should be addressed to Dr. Jeff W. Lichtman, Department of Anatomy and Neurobiology, Box 8108, Washington University School of Medicine, 660 South Euclid, St. Louis, MO 63110. E-mail: jeff@thalamus.wustl.edu.

Dr. Marques's present address: Departamento de Anatomia, Instituto de Biologia, Universidade Estadual de Campinas, UNICAMP, Campinas Sao Paulo, Brasil 13083-970. E-mail: marques@obelix.unicamp.br.

Dr. Conchello's present address: Institute of Biomedical Computing, Washington University, Old Shriner's 2229, Box 8036, St. Louis, MO 63110.

Copyright (C) 2000 Society for Neuroscience $\quad 0270-6474 / 00 / 203663-13 \$ 15.00 / 0$ axis of the muscle fiber were seen in developing gutters, although the orientation of the gutters seemed to be imposed by the branching pattern of the nerve. Eventually, superficial regions lacking AChR labeling were apparent in all junctions. In junctions denervated in the neonatal period both gutter formation and the disappearance of superficial receptors regions were prevented. We suggest that tension between growing muscle fibers and the relatively inelastic synaptic terminals that adhere to them causes the topographic features of the postsynaptic membrane. This view provides a mechanical explanation for gutters, folds, and the location of folds at sites of neurotransmitter release.

Key words: reflected light confocal microscopy; postsynaptic membrane topography; acetylcholine receptor distribution; neuromuscular junction development; synapse elimination; active zones elaboration of folds within the gutters could not be related to the changes occurring in the location of AChRs.

Techniques that are useful for studying three-dimensional topography of synapses, such as scanning (Fahim et al., 1983; Desaki and Uehara, 1987) and transmission (Teraväinen, 1968; Kelly and Zacks, 1969; Bennett and Pettigrew, 1974; MatthewsBellinger and Salpeter, 1983) electron microscopy, are not ideal for assaying changes in AChR distribution. On the other hand, fluorescence techniques useful for the study of receptor distribution do not have the resolution to permit analysis of threedimensional topography. In the present work a technique is described in which biotin-bungarotoxin bound to avidinhorseradish peroxidase (HRP) is used to deposit a highly reflective substance on the AChRs of the neuromuscular junction. By using reflected light confocal microscopy to generate threedimensional image reconstructions, we were able to obtain a highly resolved topography of the AChR-rich region that exceeded the resolution of fluorescence imaging. With this new approach the gutters, the folds, and the overall AChR distribution could all be viewed at the same time throughout development.

\section{MATERIALS AND METHODS}

Animals and muscle preparation. Neonatal mice were obtained from breeding colonies established in our animal care facility. Pregnant females were isolated and checked daily. The date of birth was designated postnatal day $0(\mathrm{P} 0)$, and the pups were weaned on P21. Embryos [embryonic day 15 (E15) and E19] were obtained from timed pregnant females (CF1B strain, Harlan Sprague Dawley, Indianapolis, IN), and their prenatal age was confirmed from external physical characteristics. Forty animals (six adults and 34 young animals ranging in age from E15-E20 postpartum) were used. 

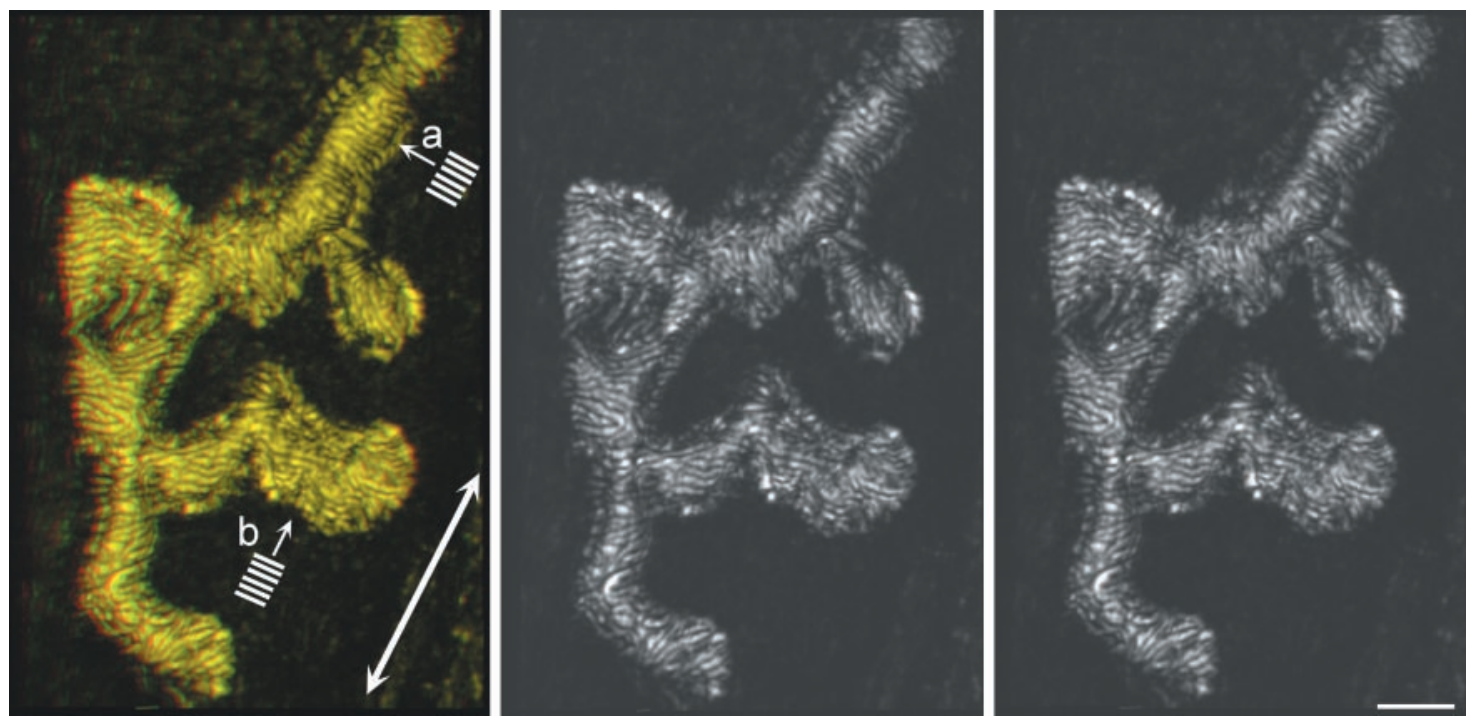

Figure 1. Visualizing the three-dimensional topography of the AChR distribution in the postsynaptic membrane of an adult mouse neuromuscular junction. Left, Red/green overlay of the three-dimensional topography of the AChR-rich neuromuscular junction (for viewing with red, the right eye, and for viewing with green, the left eye glasses). Middle, Right, Stereo pairs (for crossed eye viewing: left eye views right image) of the same junction (note that subsequent three-dimensional figures are all displayed this same way). In this reconstruction the depth of the junctional gutter (going into the plane of the page) and the pattern of alternating bright and dark bands are visible (especially at the bottom of the gutters). Orientation of the folds shows a tendency that they be orthogonal to the long axis of the muscle fiber (doubleheaded arrow). Thus gutters coursing parallel to the long axis of the muscle fiber have folds that generally run transversely $(a)$, whereas branches that run perpendicular to the long axis often have folds that run parallel to the long axis of the gutter $(b)$. Scale bar, $4 \mu \mathrm{m}$.

Animals were anesthetized by an intraperitoneal injection of sodium pentobarbital, and the muscle was fixed. The protocol for fixation involved cardiac perfusion with Ringer's solution, followed by cold fixative freshly prepared $(2 \%$ paraformaldehyde and $0.1 \%$ glutaraldehyde in PBS, $\mathrm{pH}$ 7.4). Embryos were removed from the mother after fixation. Both sternomastoid muscles were removed, pinned out on Sylgard-coated (Dow, Midland, MI) dishes, and washed for 3-4 min with Ringer's solution. In some animals the sternomastoid muscle was denervated on P10 by cutting the muscle nerve; $7 \mathrm{~d}$ later the animals were killed, and the muscles were removed as above.

$A C h R$ staining. Muscles were incubated with biotin- $\alpha$-bungarotoxin (b-BTX; $1 \mu \mathrm{g} / \mathrm{ml}$; Molecular Probes, Eugene, OR) for $4 \mathrm{hr}$ at room temperature and then extensively washed with Ringer's solution (Baxter, Deerfield, IL). After that, they were incubated overnight with avidinHRP (a-HRP; $1 \mu \mathrm{g} / \mathrm{ml}$; Molecular Probes). A dark gray HRP reaction product was deposited by using the Vector SG kit (Vector Laboratories, Burlingame, CA). Then the muscles were washed in cold $30 \%$ ethanol for 4 min, dehydrated, and mounted in Permount. In some muscles, AChRs were tagged fluorescently with tetramethyl-rhodamine-bungarotoxin (rhBTX; $5 \mu \mathrm{g} / \mathrm{ml}$; Molecular Probes) for $30 \mathrm{~min}$ at room temperature, washed with Ringer's, and mounted in Vectashield Mounting Medium (Vector Laboratories) to lessen bleaching.

Frog neuromuscular junctions also were stained and observed with confocal reflected light. Frogs were cold-anesthetized and pithed. Cutaneous pectoris and sartorius muscles were dissected out from Rana pipiens in Ringer's-Frog solution and pinned out in a Sylgard Petri dish. The muscles were incubated in b-BTX $(1 \mu \mathrm{g} / \mathrm{ml}$; Molecular Probes) for $4 \mathrm{hr}$ at room temperature and after a washing in PBS incubated in a-HRP $\left(1 \mu \mathrm{g} / \mathrm{ml}\right.$; Molecular Probes) overnight at $4^{\circ} \mathrm{C}$. After several washes with Ringer's-frog, HRP was visualized by using the Vector SG kit. Muscles were fixed with a cold solution of $2 \%$ paraformaldehyde and $0.1 \%$ glutaraldehyde in PBS for $1 \mathrm{hr}$, washed with PBS for 4-5 min, dehydrated, and mounted in Permount for observation. The mouse and frog preparations maintained their AChR labeling for $>1$ year.

Confocal microscopy. Muscles were examined with the reflected light port of a laser-scanning confocal microscope (Odyssey, Noran Instruments, Middleton, WI) linked to an upright microscope (Optiphot-2, Nikon, Tokyo, Japan), using a $100 \times, 1.4$ numerical aperture Plan Apo oil objective (Nikon). The excitation source was the 528.7 line of an argonion laser. Reflected light confocal used a small pinhole aperture (diameter of $0.1 \mu \mathrm{m}$ in specimen space). Optical sections were collected at 0.1 $\mu \mathrm{m}$ intervals, and the number of images in the z-plane varied from 40 to
80. The stacks of images were processed into stereo pairs or movies (using a maximum voxel ray tracing algorithm) with locally modified volume-rendering software on a Sun computer (Sparc 370 with TAAC board). An index of junctional area was measured by taking the product of the maximum length of the junction in the long axis of the muscle fiber and the maximum width of the junction in the orthogonal axis.

\section{RESULTS}

\section{Reflected light confocal microscopy: Theory}

The principal aim of this work was to study the development of the postsynaptic membrane at the neuromuscular junction. We were interested in using a technique that would highlight the location of AChRs in the postsynaptic membrane, because a number of lines of evidence suggest that the distribution of AChRs undergoes major changes during early development. We also wanted a technique that provided topographic (threedimensional) views of the postsynaptic membrane.

We took advantage of the high refractive index of the reaction product of HRP deposited at sites containing AChRs (see Materials and Methods), which allowed for visualization with epiillumination reflected light (i.e., the microscope objective was used both to illuminate and view the specimen). With this technique the postsynaptic membrane of adult mouse neuromuscular junctions shows a bright reflection (Fig. 1). The reflection at high magnification and with high numerical aperture objectives showed alternating bright and dark bands reminiscent of SEM images of postsynaptic folds (see, for example, Fahim et al., 1983; Ogata and Yamasaki, 1985; Desaki and Uehara, 1987).

In distinction to the many points of scattered light induced by HRP reaction product seen in retrogradely HRP-labeled cells viewed in dark field (see, for example, Lichtman et al., 1984), the reflection seen after labeling of the AChRs at the adult neuromuscular junction appeared specular (i.e., mirror-like). Such reflections occur when reflecting surfaces are continuous over distances that are greater than the wavelength of the reflecting light. 

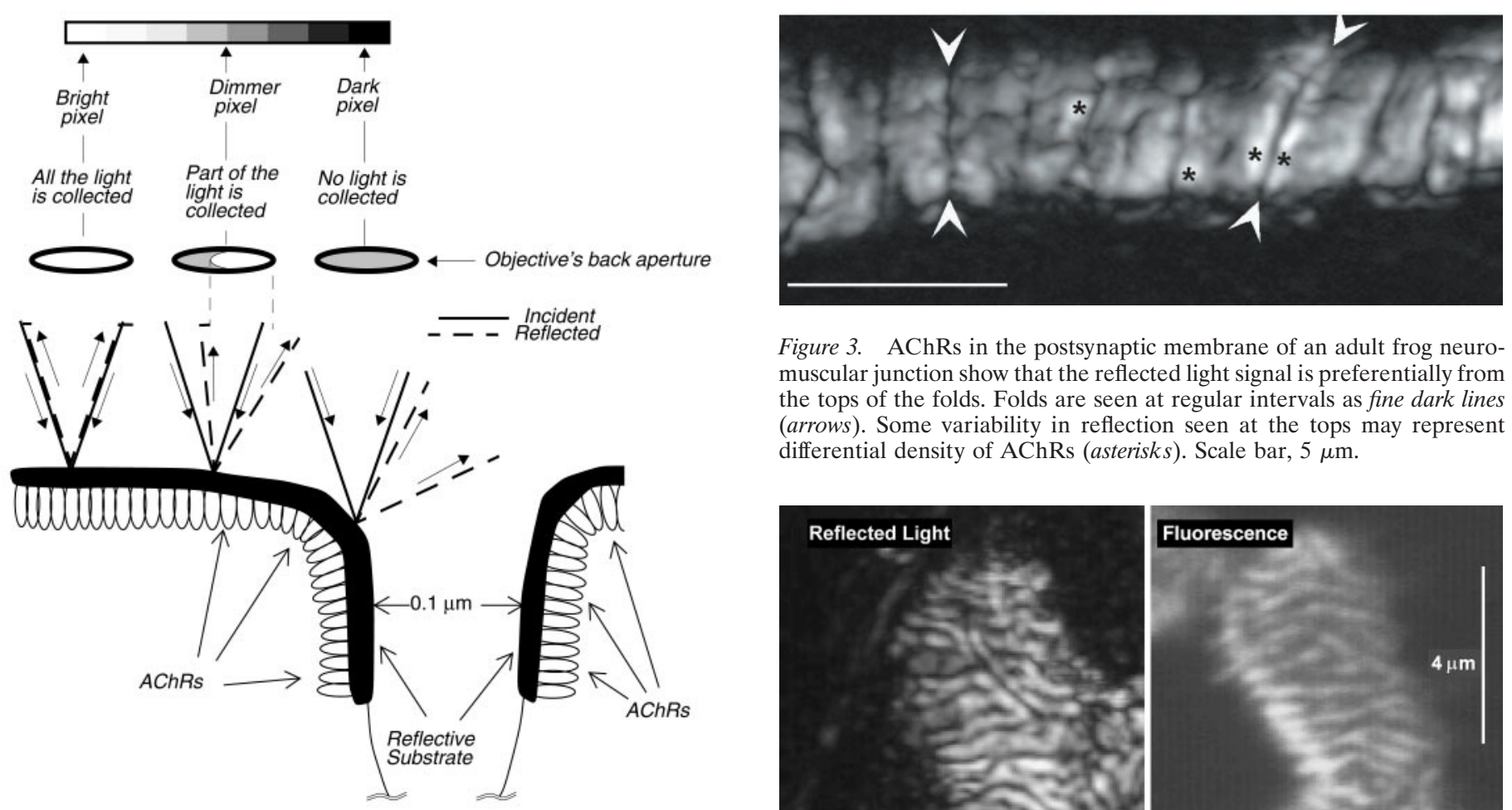

Figure 3. AChRs in the postsynaptic membrane of an adult frog neuromuscular junction show that the reflected light signal is preferentially from the tops of the folds. Folds are seen at regular intervals as fine dark lines (arrows). Some variability in reflection seen at the tops may represent differential density of AChRs (asterisks). Scale bar, $5 \mu \mathrm{m}$.
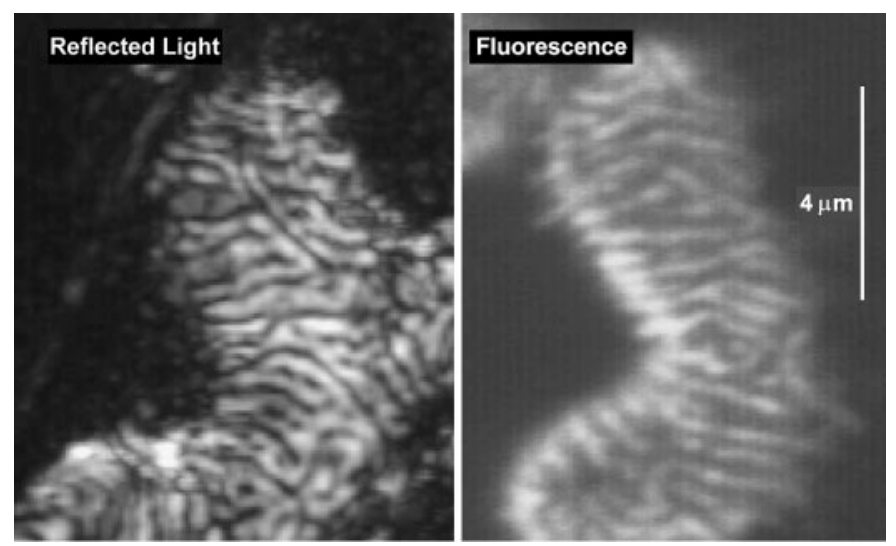

Figure 4. Comparison of fluorescence and reflected light confocal images of postsynaptic receptor labeling. AChRs in the postsynaptic membrane of two different adult mouse neuromuscular junctions were labeled for reflected light (left) or fluorescence (right). Better resolution of the folds was achieved with reflected light because of the orientation dependence of the reflection. A second difference is that the folds do not reflect light (and appear as dark bands), whereas they are highlighted preferentially in fluorescence. Conversely, the tops of the folds are bright in reflected light but relatively dim in fluorescence imaging.

A specular reflection obeys Snell's law such that the angle of each incident ray will be coplanar, equal, and opposite the angle of each reflected ray. If the reflection from the neuromuscular junction is specular, the light collected by the microscope objective should be critically dependent on the tilt of each part of the postsynaptic membrane. In particular, light should be collected most efficiently from the parts of the receptor-rich membrane that are oriented orthogonal to the optical axis. It is known that AChRs are located both at the tops of the folds and part way down the sides (Flucher and Daniels, 1989). Thus in observing a postsynaptic fold from above the tops of the folds, we should see more light reflected back into the objective than from the infolded regions (Fig. 2, top). Therefore, membrane regions oriented at progressively more extreme angles should return correspondingly less light through the objective. The folds are but a small part of the entire postsynaptic topography, which is arranged in large gutter-shaped troughs (Fig. 2, bottom). The folds on the sides of the gutter will be angled such that they return less light than those at the bottom of the gutter, in part because of stacks of multiple reflecting surfaces that prevent efficient illumination and detection.

These expectations were borne out (see Fig. 1). The bottom and the top of the gutter (both orthogonal to the optical axis) 

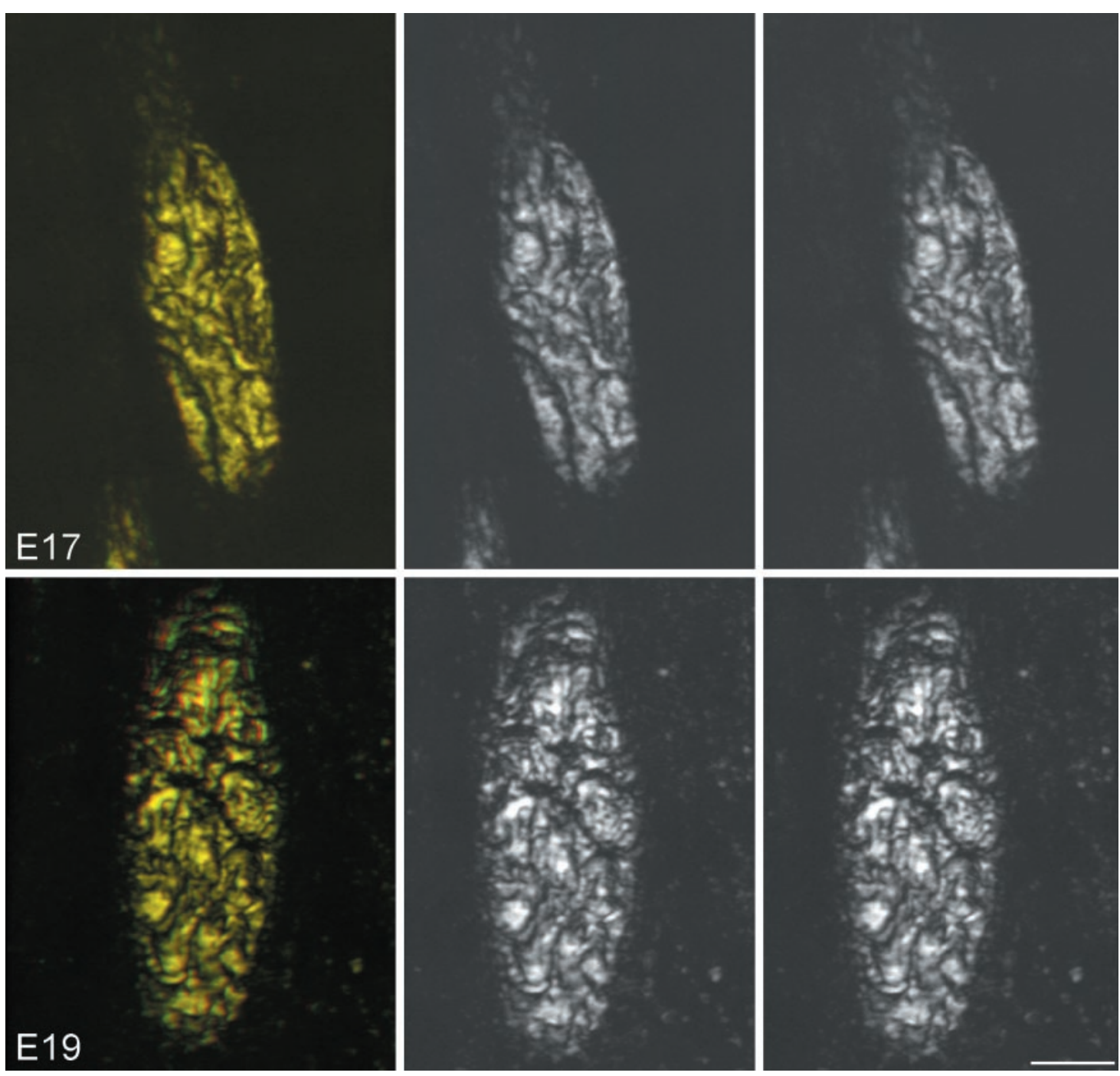

Figure 5. AChR distribution at neuromuscular junctions in the late embryonic period, E17 and E19. Before birth the postsynaptic AChR plaque on each muscle fiber is typically oval. Often the plaque is indented relative to the muscle fiber membrane. Within the plaque, patches of AChRs are arranged as cobblestones (2-3 $\mu \mathrm{m}$ in diameter) separated by dark bands that may represent nascent folding of the postsynaptic membrane. Left panels, Red/green stereo pair. Middle, Right panels, Stereo pairs (left eye views right image). Scale bar, $4 \mu \mathrm{m}$.

were typically more reflective than the sides. In addition, the alternation of bright and dark bands seemed to be associated with the finer grain topography of the folds; these bands had a spatial frequency of $\sim 1.6$ dark bands/ $\mu \mathrm{m}$, which is in line with the frequency of folds from electron microscopic studies of the sternomastoid (Salpeter, 1987). However, because the widths of the tops and infoldings are somewhat similar in mammals (see, for

\section{Table 1. Changes in AChR area of the neuromuscular junction during} postnatal development

\begin{tabular}{ll} 
Age & Area $\left(\mu \mathrm{m}^{2}\right)$ \\
\hline P01 & $138.0 \pm 52.6$ \\
P04 & $173.3 \pm 20.2\left(^{*}\right)$ \\
P06 & $279.0 \pm 50.0$ \\
P08 & $238.0 \pm 36.6\left(^{*}\right)$ \\
P10 & $430.3 \pm 33.3\left({ }^{*}\right)$ \\
P15 & $719.9 \pm 57.5$
\end{tabular}

Values reported are mean $\pm \mathrm{SD}(n=5$ endplates/age). *Significantly different from the next age value ( $p<0.05$; Student's $t$ test). example, Padykula and Gautier, 1970), we could not be sure whether the bright reflections were from the tops or infolded regions. To determine whether it was the tops of folds that were the source of the bright reflecting bars (as should be the case if the reflection were specular), we viewed the AChRs in the postsynaptic membrane of frog neuromuscular junctions. Frog junctions have folds that typically are spaced at regular intervals, with the interfold regions being (unlike mammalian junctions) substantially longer than the width of the folds themselves. We observed that the reflected light image of frog postsynaptic membrane (Fig. 3) showed wide areas that reflected light, interrupted by fine dark lines at regular intervals (Fig. 3, arrows), confirming that the tops of the folds were reflecting light preferentially. Interestingly, the brightly reflecting regions showed some variability in reflection (Fig. 3, asterisks), which may be related to a higher density of AChRs on the tops of folds described previously (Shotton et al., 1979).

Close inspection of images of the postsynaptic membrane of mammalian junctions also supported the idea that the tops of the folds were reflecting light preferentially. We found, for example, 

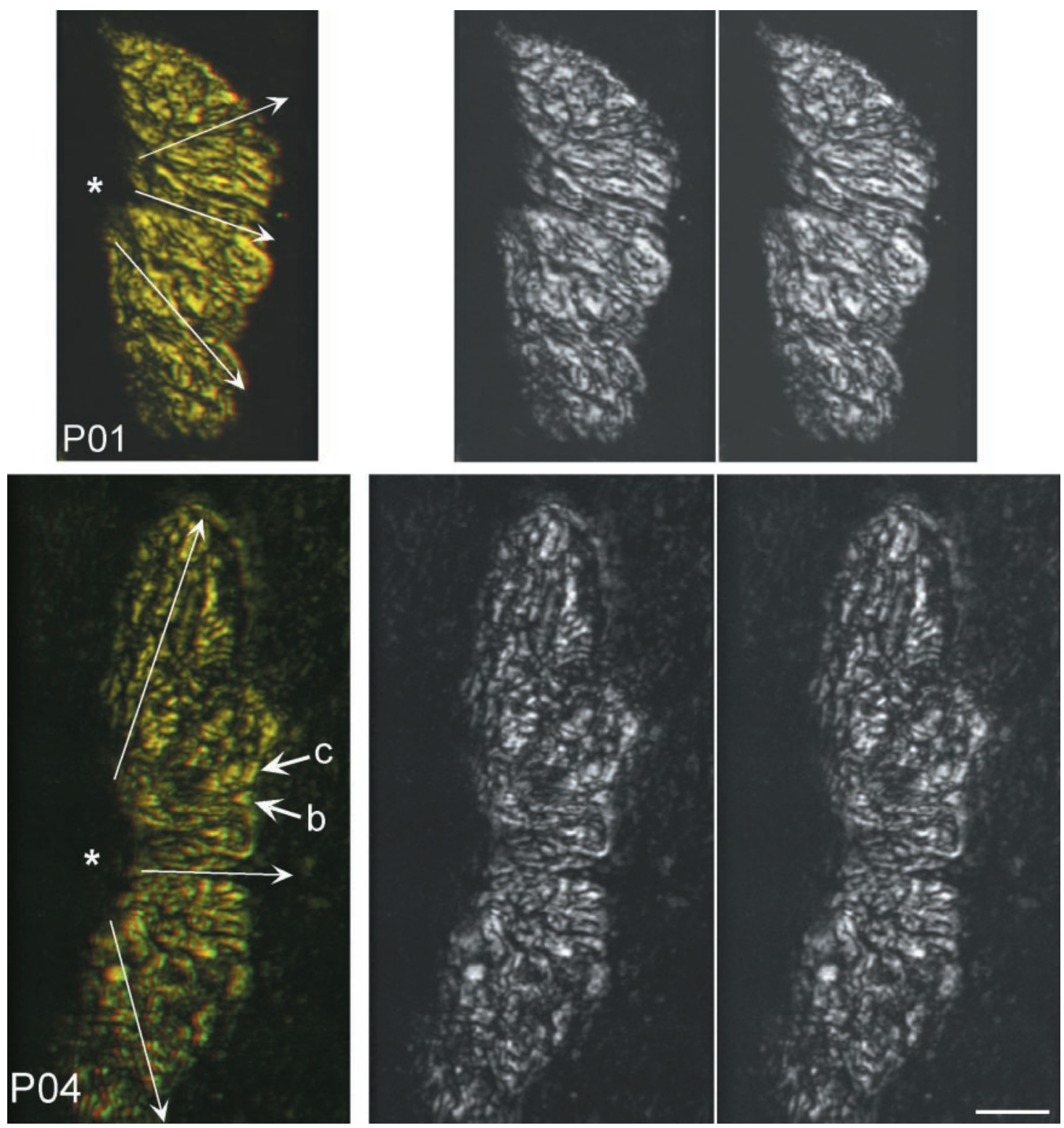

Figure 6. AChR distribution at the neuromuscular junction during the early postnatal period, P01 and P04. At birth (P01) AChRs were distributed in many small elongated regions that had a radial pattern from one point in the endplate, which we presume is the point of nerve entry $\left({ }^{*}\right)$. At later periods (P04) AChRs started to show a topographical segregation so that coexisting within the same junction were AChRs in creases $(c)$ and in bulges $(b)$. Left panels, Red/green stereo pair. Middle, Right panels, Stereo pairs (left eye views right image). Scale bar, $4 \mu \mathrm{m}$.

that the dark bands were all approximately the same width (at the diffraction limit, $\sim 0.2 \mu \mathrm{m})$, whereas the bright reflecting bands were wider and more variable in width $(0.5-1 \mu \mathrm{m}$, on average) (Fig. 4, left). This is consistent with the electron microscopic data indicating that the width of the fold is relatively constant and below $0.2 \mu \mathrm{m}$, whereas the distance between folds can vary (Salpeter, 1987).

A comparison of fluorescently labeled and reflected light images of AChRs in the postsynaptic membrane showed the advantages of reflected light imaging (Fig. 4). We found that the orientation dependence of the reflection allowed the reflected images to have greater contrast than fluorescence images of comparable samples. Because fluorescence emission is isotropic, part of the fluorescence signal is always directed into the objective irrespective of the orientation of the labeled membrane. In addition, with fluorescence, regions of the membrane at steep angles give greater signals because the fluorescence from multiple depths adds together. Thus the infolded region (which, because of its steep angle, appears dark in reflected light) is the brightest part of the image with fluorescence because nearby AChR-rich membrane regions sum. This summing cannot be ameliorated by confocal microscopy, because the optical depth of focus even with confocal $(\sim 0.75 \mu \mathrm{m})$ is much wider than the thickness of a membrane so that the intensity of the fluorescence signal is going to be brighter for any membrane that is tilted.

\section{Features of the adult neuromuscular junction}

The reconstructions of adult mouse neuromuscular junctions showed an unexpected relation between the orientation of the folds and the orientation of the muscle fiber. In particular, folds tended to run transverse to the long axis of the muscle fiber. Thus when the gutters were running parallel to the long axis of the 

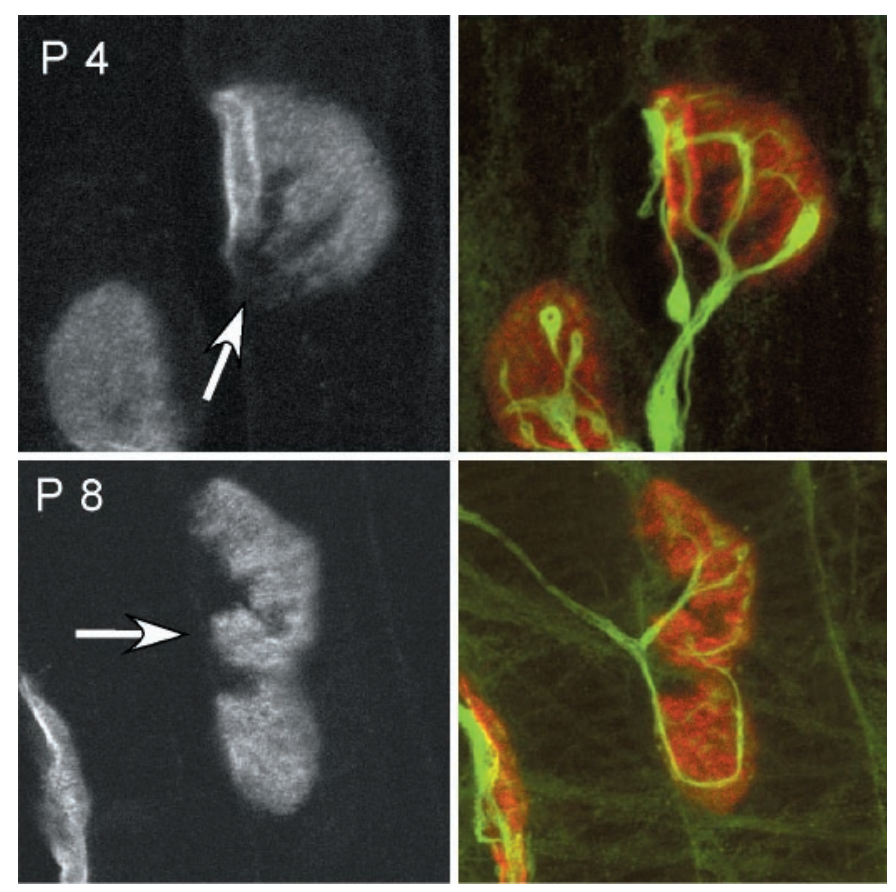

Figure 7. Site of nerve entry associated with frayed appearance of AChRs in the postsynaptic membrane. The left panels show the AChR distribution labeled with rhodamine-tagged $\alpha$-bungarotoxin of a P4 (top) and P8 (bottom) neuromuscular junction from the sternomastoid muscle. In each case the junctional edge is uneven on one side (arrows). The right panels show nerve terminal staining with neurofilament antibody (green) superimposed on the receptor labeling (red). In these two junctions, as is invariably the case, the nerve terminal entry direction corresponds with the uneven receptor staining (compare the axon entry in each junction with the arrows in the left panels). The P4 neuromuscular junction is multiply innervated by a large and a small caliber axon. We have observed that the multiple axons always enter the junction from the same side.

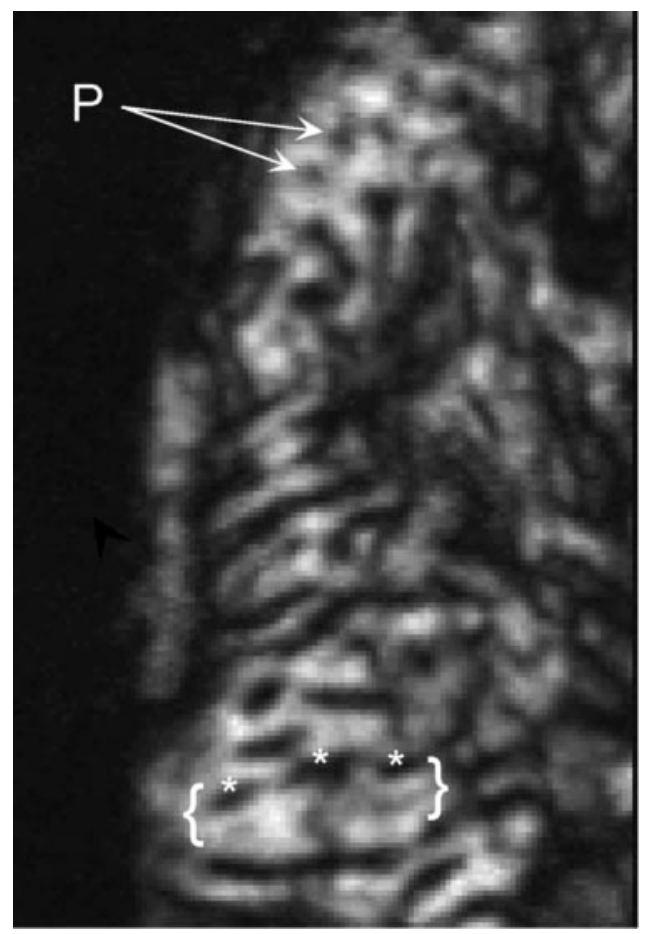

Figure 9. High-magnification image of a P06 neuromuscular junction suggests the steps in fold formation. At this age small dark pits $(P)$ are seen. In addition, oblong dark pits appear to be coalescing into contiguous folds (asterisks). At some sites in which AChR reflection is high there is an unusually large distance between adjacent folds (brackets). Because such large interfold distances are rarely seen at adult mammalian neuromuscular junctions, these sites presumably will undergo fold formation soon. Indeed, a faint, dim broken line sometimes was observed in the middle of such interfold regions, which may be the earliest stage in fold formation.
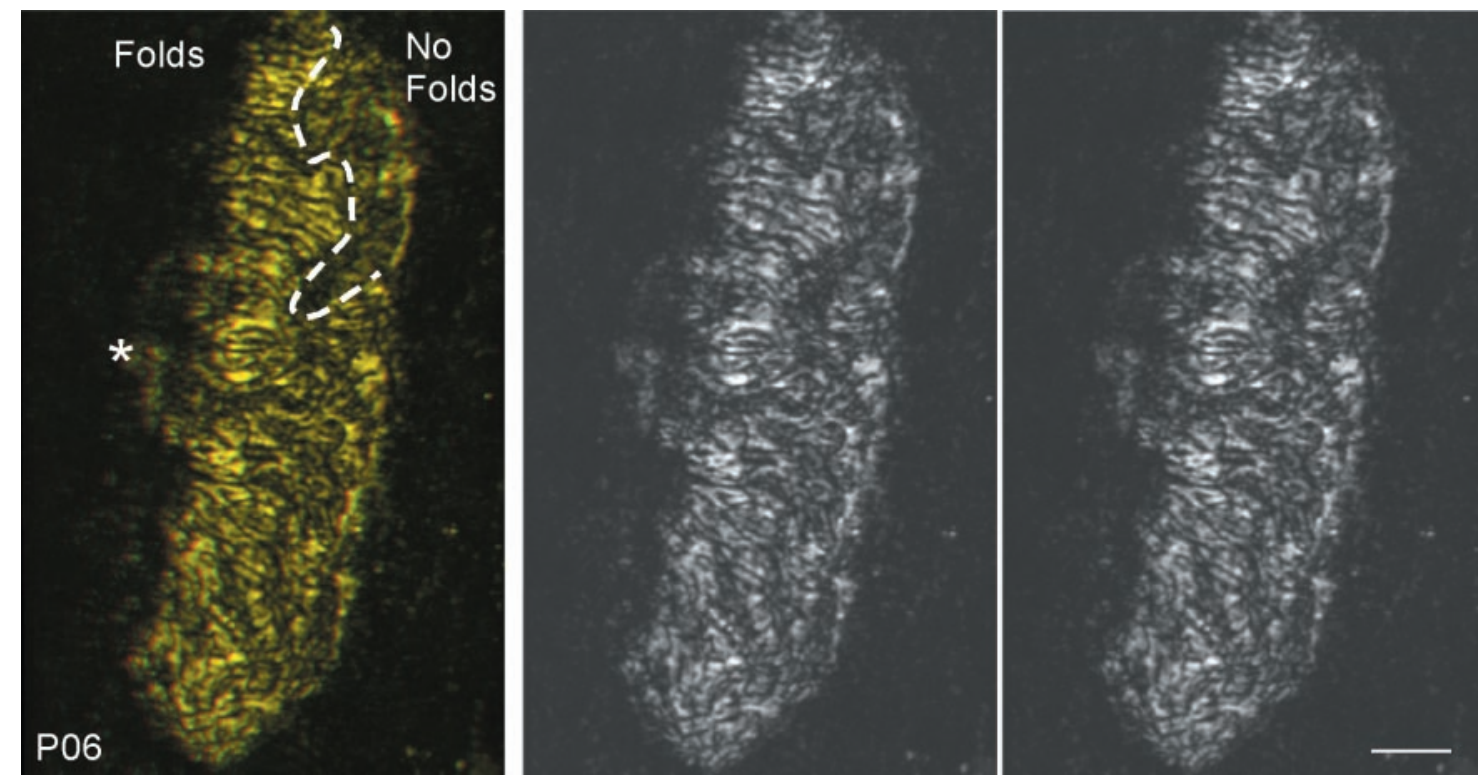

Figure 8. AChR distribution at the end of the first postnatal week, P06. At many neuromuscular junctions the topographical areas of the postsynaptic membrane become segregated into two well defined categories; some parts of the receptor pattern show organized folding, whereas other regions have more disorganized and less intensely reflecting receptor labeling. The smoothness of the edges is more obvious. Based on previous work (Balice-Gordon and Lichtman, 1993), in this junction the nerve is probably entering from the left (asterisk). Left panels, Red/green stereo pair. Middle, Right panels, Stereo pairs (left eye views right image). Scale bar, $4 \mu \mathrm{m}$. 

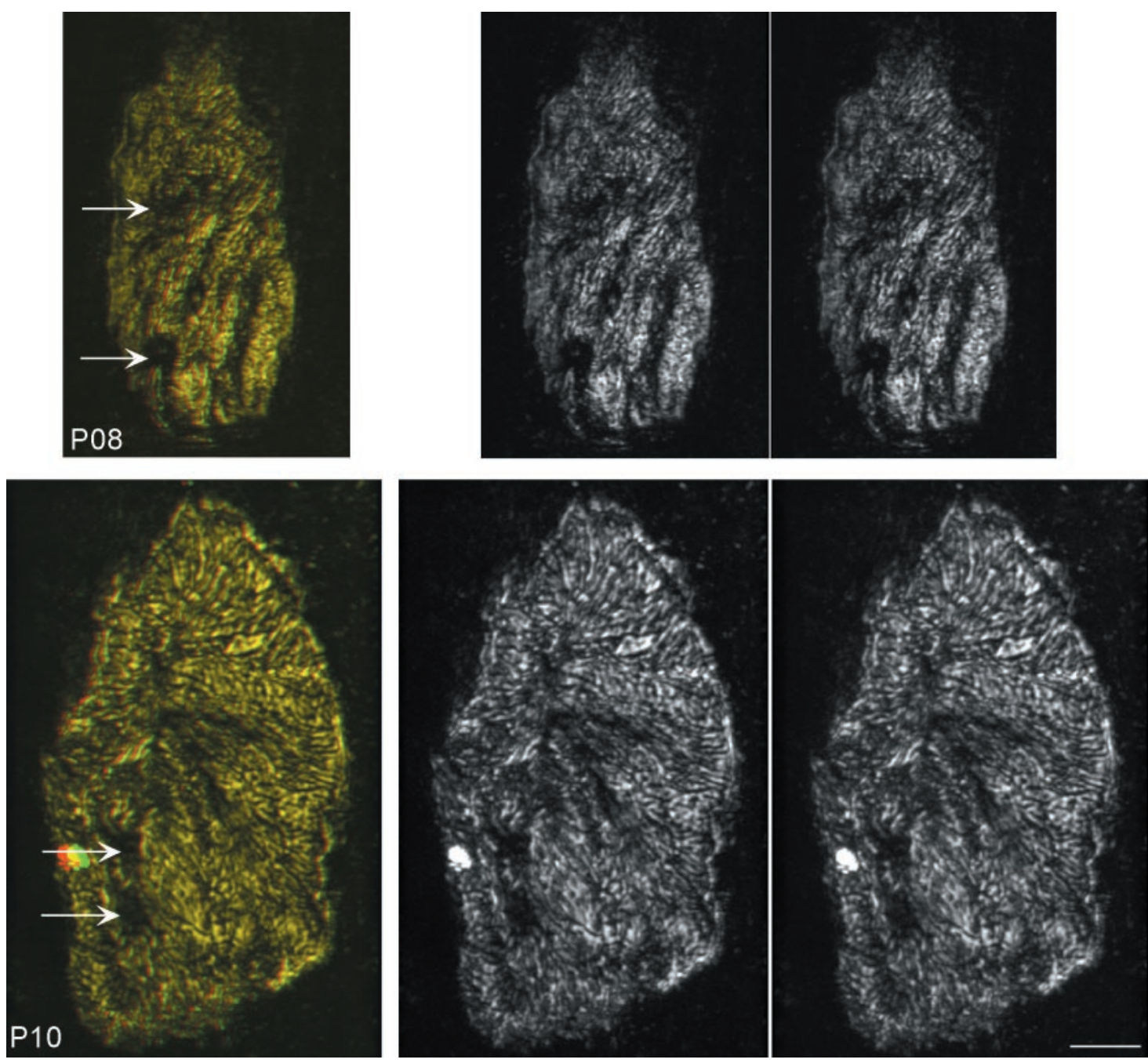

Figure 10. AChR distribution at neuromuscular junctions at the beginning of the second postnatal week, P08-P10. By the second postnatal week it was common to see receptor-rich and receptor-poor areas within the neuromuscular junction. The two endplates shown here, still mainly oval, represent the early stages of this process. Areas lacking reflection appear as small holes and strips of different sizes (arrows). With subsequent growth the receptor-rich areas increase in area, and the size of the receptor-poor areas also increases. Left panels, Red/green stereo pair. Middle, Right panels, Stereo pairs (left eye views right image). Scale bar, $4 \mu \mathrm{m}$.

muscle fiber, the folds were always transverse to the length of the gutters (see Fig. 1, $a$; 19 branches in 10 junctions), but when the gutters ran circumferentially, the folds tended to be parallel with the long axis of the gutters (see Fig. 1, b; 10 of 12 branches in six junctions). This relation indicates a role for the muscle fiber in establishing the folding pattern and suggests a mechanism that could lead to fold formation (see Discussion).

\section{Neuromuscular junction development}

By taking multiple reflected light confocal optical sections and reconstructing them, we obtained views of the three-dimensional topography of the receptor-rich regions of the postsynaptic membrane. Approximately 740 neuromuscular junctions from 80 sternomastoid muscles in mice ranging in age from E17-P15 from were studied. Forty-two representative examples were chosen for detailed analysis and reconstruction.

Previous work has shown that there is variability between junctions during early postnatal life because of a developmental "wave," such that junctions at several different stages of maturation coexist at the same time (Balice-Gordon and Lichtman, 1993; Colman et al., 1997; Gan and Lichtman, 1998). It is thus difficult to establish unambiguous evidence for the steps in the dynamic process with techniques that provide only a single time point snapshot of any particular cellular element. Thus far, however, we have been unable to use reflected light to see the maturation of the neuromuscular junction in living animals. Despite this caveat, we have observed a number of morphological features, detailed below, that change as a function of age.

\section{Embryonic period (E17-E19)}

The receptor aggregates seen on fibers of 17-d-old embryos appeared as loose accumulations of small patches of receptors, and three-dimensional reconstructions showed that they were mainly in one plane (Fig. 5). We found, as expected, that in the late embryonic period the postsynaptic $\mathrm{AChR}$ plaques were oval or round. The reconstructions indicated that these plaques were slightly concave relative to the nearby muscle fiber membrane, typically in the shape of a spoon (see Discussion). Within the plaque the receptors were arranged in patches that resembled cobblestones (2-3 $\mu \mathrm{m}$ in diameter) that were separated by well defined dark bands, presumably because of nascent folding of the postsynaptic membrane (Fig. 5). 

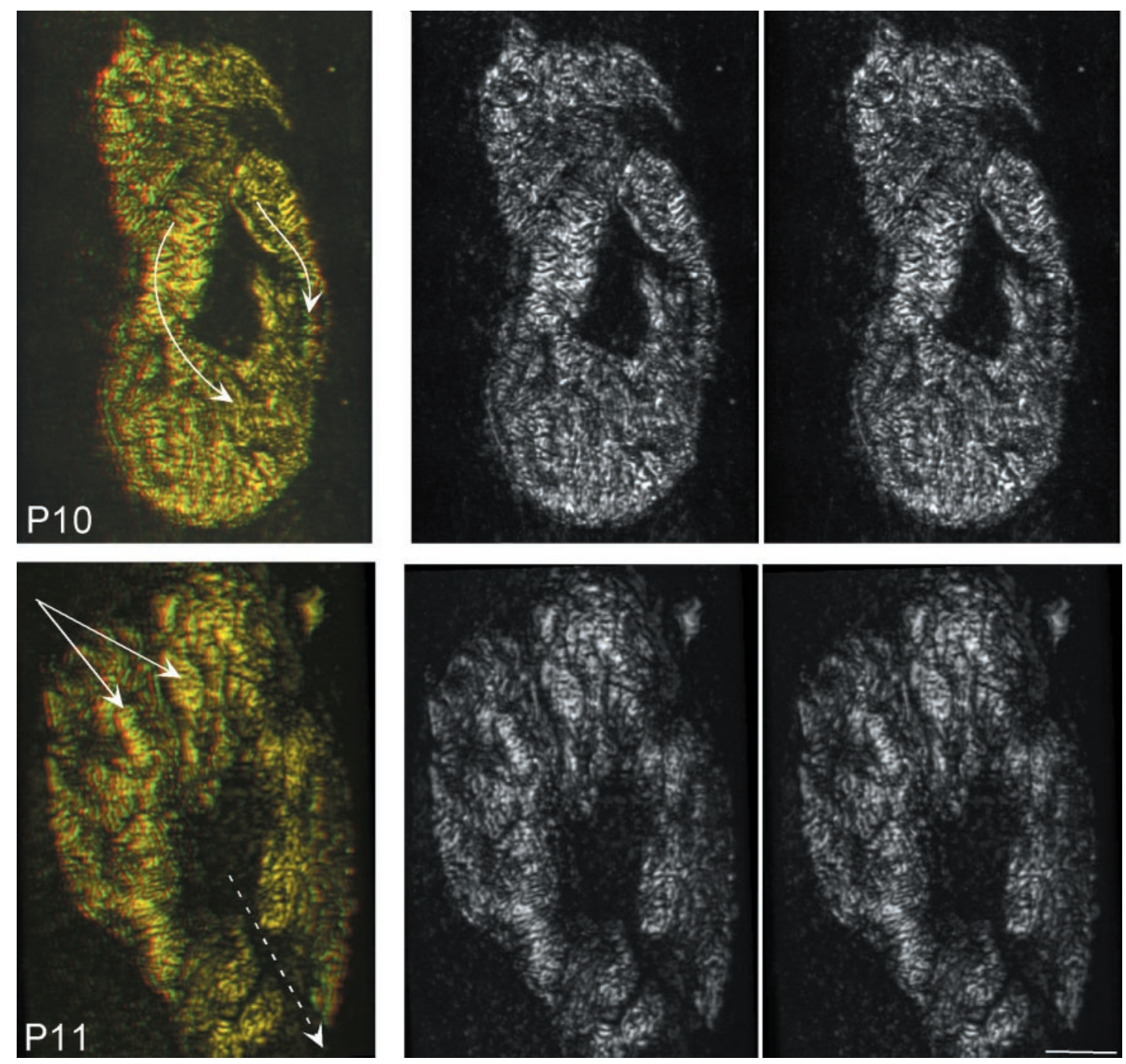

Figure 11. AChR distribution at neuromuscular junctions during the middle of the second postnatal week, P10-P11. Continued loss of postsynaptic sites causes perforations in the receptor density to enlarge and coalesce. Gutters containing well developed folds (presumably associated with nerve branches) are quite obvious (arrows). Areas that lose AChRs are superficial and appear as crests between adjacent deeper gutters. At the neuromuscular junction shown from P10, the bottom part of the junction appears to be terraced with receptor staining at two or more distinct depth levels. At the endplate from P11 it is clear the idea that the loss of AChR staining sculpts the outside edges, and these points are connected to the interior holes by channels lacking reflection (dashed arrow). Left panels, Red/green stereo pair. Middle, Right panels, Stereo pairs (left eye views right image). Scale bar, $4 \mu \mathrm{m}$.

\section{Early postnatal period (P01-P04)}

At birth, all of the endplates studied were still oval. However, with regard to the membrane topography, some junctions resembled embryonic junctions $(\sim 40 \% ; n=60)$, whereas others showed more complexity. One trend was that the AChR-positive regions tended to break apart into smaller regions than seen at earlier ages. Rather than appearing as cobblestones, in most junctions during the first few postnatal days the receptors were grouped into hundreds of small elongated patches. The elongated receptor patches were aligned approximately in a radial pattern from one point near the side of the junction (Fig. 6, arrows), which may be related to the point of nerve entry. At most junctions the continuous outline of the plaque was interrupted by indentations at one of its edges, leading for the first time to obvious asymmetry in the overall shape. Given that in adult neuromuscular junctions the site of nerve entry contains few AChRs (see for example, BaliceGordon and Lichtman, 1993), the early developing asymmetry probably also is related to the fact that the nerve entry is from one side. Indeed, in the neonatal period the receptor distribution is always frayed and missing at the nerve terminal entrance site (Fig. 7). In addition to fraying at one of its edges, the plaques also began to show an increase in topographical complexity, possessing one or more bulges and creases (see Fig. 6). At this stage the endplate resembles a crumpled blanket thrown into a shallow oblong hole.

\section{First postnatal week (P06)}

During the first postnatal week the junctions increase in area (see Table 1). In addition to the increase in length and width, the receptor-rich areas became sorted into two categories. Some parts of the plaque showed clear evidence of organized folding, whereas other regions were more disorganized and less reflective than the areas with obvious folds (Fig. 8). Three-dimensional reconstructions showed that sometimes these two kinds of receptor regions were at different depths. The sites with organized folds generally were arranged as elongated depressions or chan- 

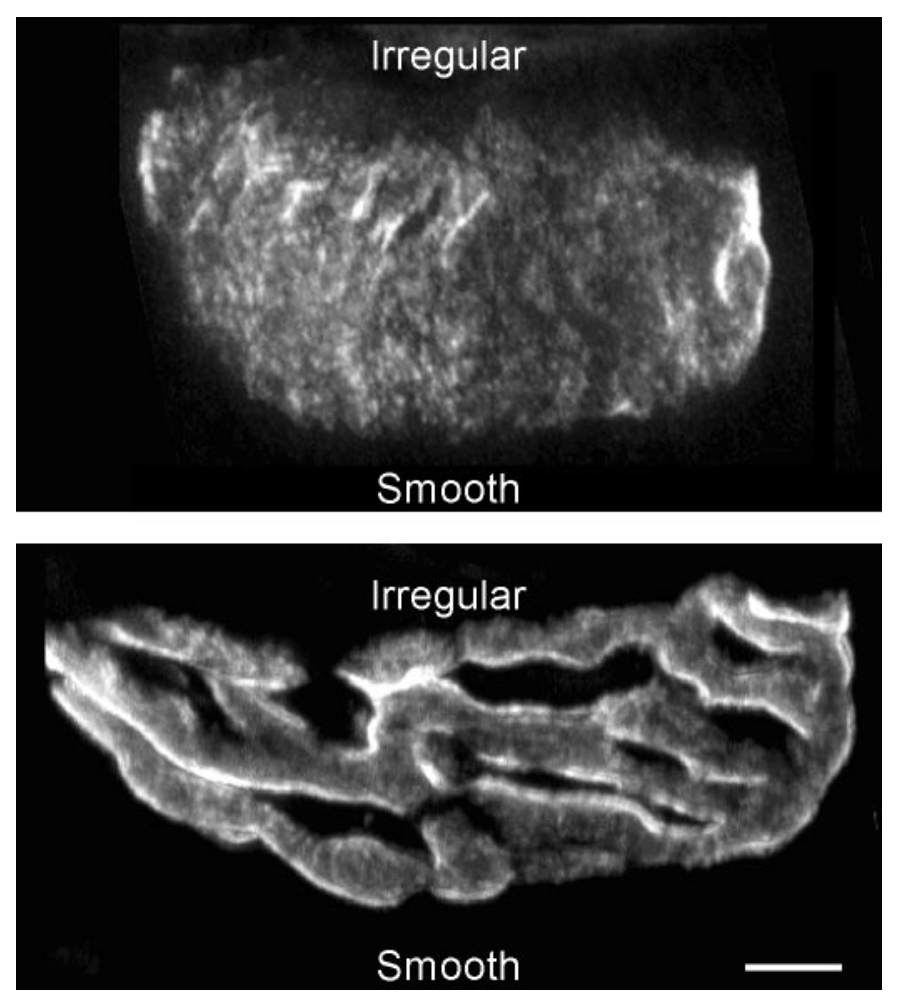

nels, suggesting that they were the precursors of the primary synaptic clefts. The disorganized receptor regions were, in contrast, located either on more superficial or in deeper regions of the muscle fiber membrane. Interestingly, in some junctions the two kinds of areas were segregated into separate contiguous regions of the neuromuscular junction (see Fig. 8, dashed line). Because competing axons segregate their synapses to separate regions during synapse elimination at this same stage (Gan and Lichtman, 1998), the differences in receptor topography and staining may be related to synapse elimination.

At many junctions the process by which organized folds become established in the deeper regions was suggested. We observed that such receptor-rich areas showed some dimpling by small dark circles (Fig. 9). It is likely that these circles represent the "pits" described by Desaki and Uehara (1987) with scanning electron microscopy. The coalescence of such pits may generate the more continuous folds eventually seen (Fig. 9, asterisks). In addition, at these ages the density of folds is sometimes lower and more

Figure 12. Asymmetry in postsynaptic AChR distribution. Shown is the rhodamine-bungarotoxin-labeled AChR distribution at a P06 (top) and P15 (bottom) neuromuscular junction. In both cases one side (lower; Smooth) maintains its smooth oval shape whereas the opposite side is fuzzier (Irregular); as suggested by numerous observations of in vivo endplates stained for both nerve and receptors, the nerve entry side is irregular (Balice-Gordon and Lichtman, 1993). Scale bar, $3 \mu \mathrm{m}$.
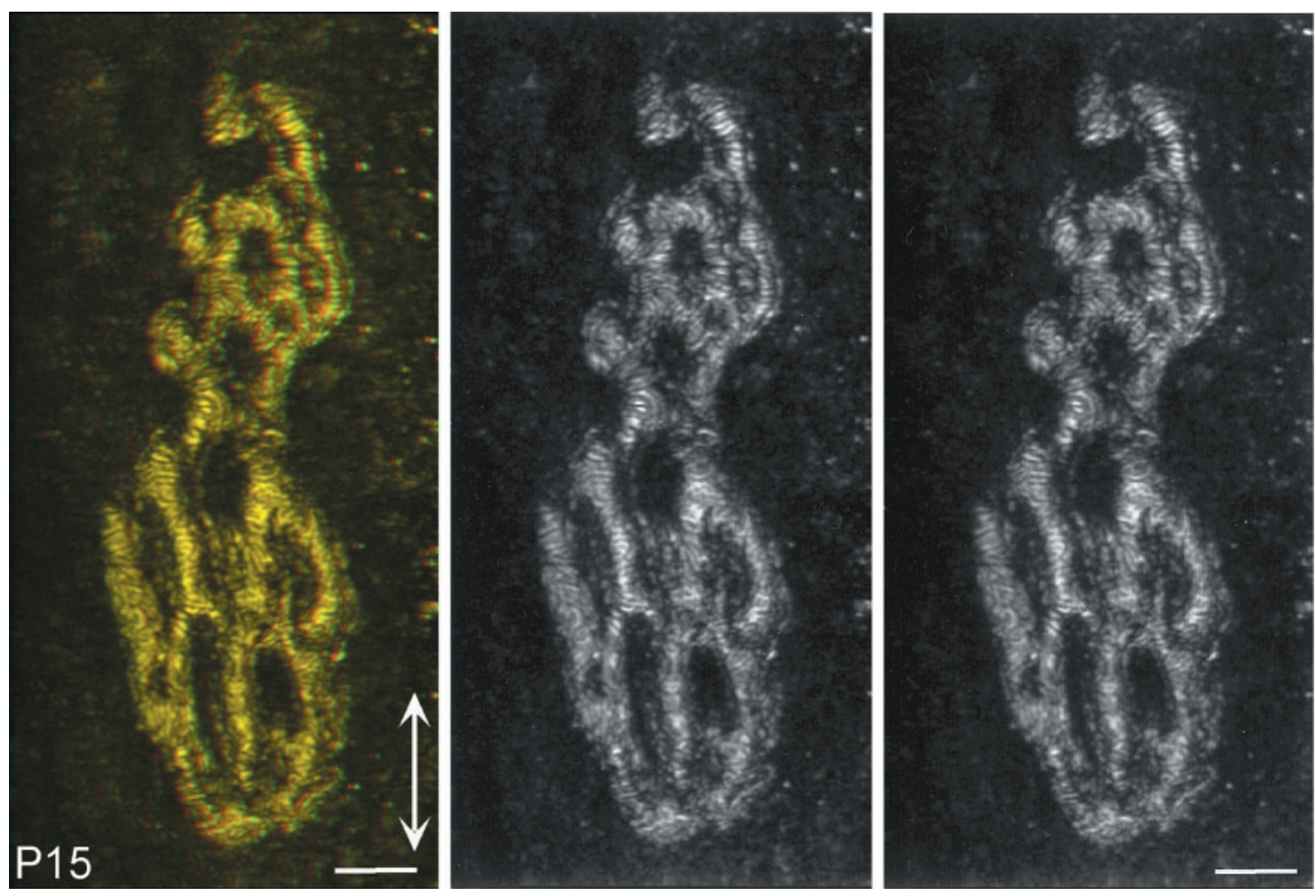

Figure 13. AChR distribution during the late second postnatal week, P15. By the end of the second postnatal week, despite their compact size, most of the endplates have attained the adult form. Well defined gutters containing receptors arranged in distinct patterns of folds are seen. Folds are oriented orthogonal to the long axis of the muscle (arrow). Because the sides of the gutter are steep, only the folds at the bottom of the gutters reflect much light back into the objective. Left panels, Red/green stereo pair. Middle, Right panels, Stereo pairs (left eye views right image). Scale bar, $4 \mu \mathrm{m}$. 

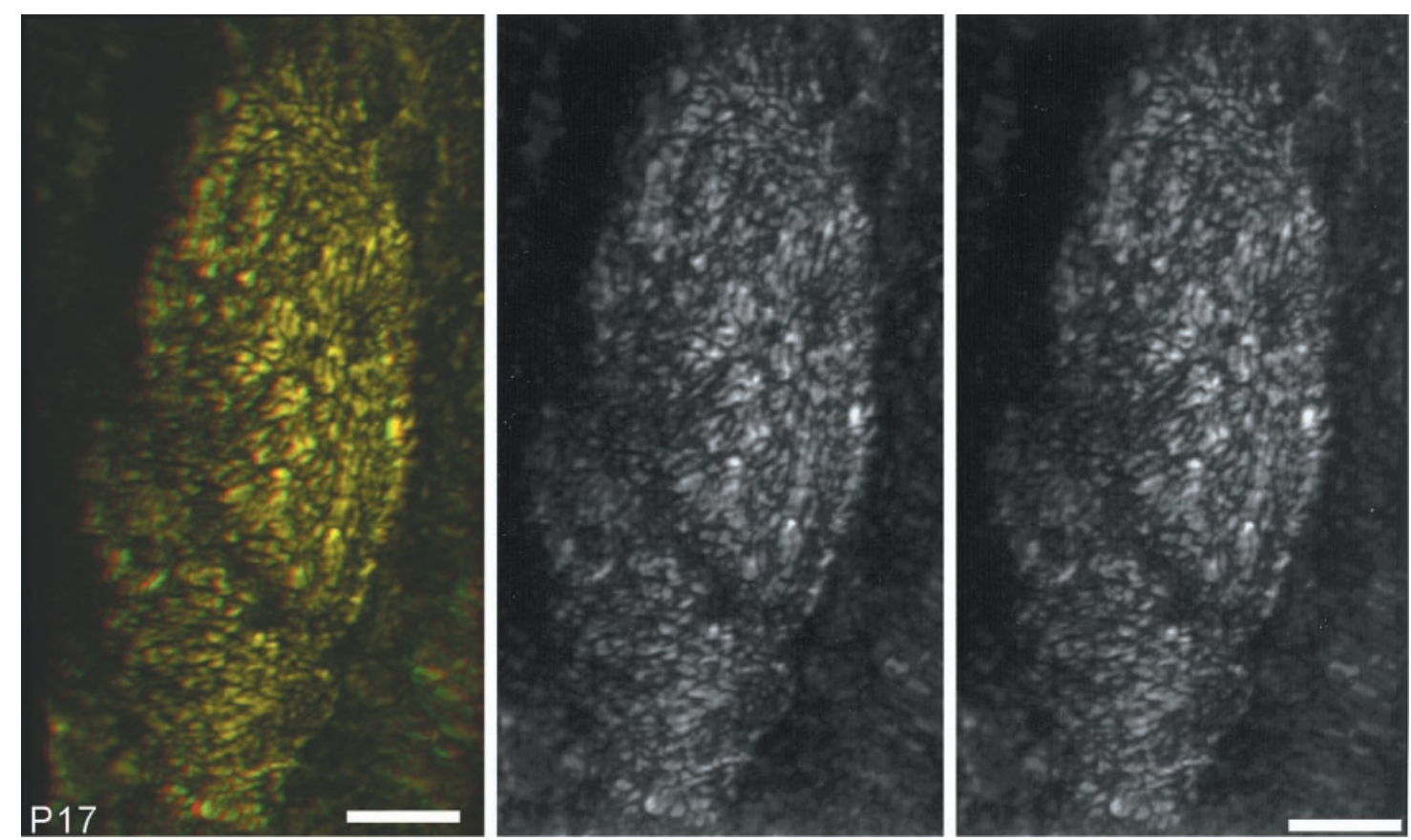

Figure 14. AChR topography and distribution do not mature at denervated neonatal neuromuscular junctions. At P17, $10 \mathrm{~d}$ after the sternomastoid muscle is denervated, the postsynaptic AChR distribution is oval, and well defined gutters are not present. Both of these features are seen commonly in junctions of much younger ages. Left panels, Red/green stereo pair. Middle, Right panels, Stereo pairs (left eye views right image). Scale bar, $4 \mu \mathrm{m}$.

"frog-like" than in adult mouse junctions (see Fig. 3), with relatively wide areas between the folds. In these interfold regions, nascent new folding is evident (Fig. 9, brackets).

\section{Second postnatal week (P08-P15)}

Measurements of endplate area (Table 1) show, as had been described previously (Balice-Gordon et al., 1993), a transient net reduction in the area of endplates at the beginning of the second postnatal week (P08). This loss of area is likely the consequence of the loss of receptor-rich regions during synapse elimination. Consistent with this loss of area, reflected light imaging of AChR staining at most endplates shows sites that lack AChR signal by the second postnatal week (Figs. 10, 11). In all junctions the loss of receptor staining not only sculpts the outside edges of the oval plaque but also removes AChRs from the interior so that one or more large regions lacking reflection are present within the junction (Fig. 11). Initially, the receptor-poor areas are small (see, for example, Fig. 10), but as junctions continue to enlarge, the sizes of the receptor-poor areas also enlarge. As the holes enlarge, they connect to the outside of the junction by extended regions lacking receptor staining (Fig. 11, bottom panels, dashed arrow). The fragmentation of the plaque into receptor-rich and receptor-poor areas gives rise to its branched appearance.

Three-dimensional reconstructions showed that the regions that lost AChR staining are (or become) superficial (Fig. 11). The gutters typically contain organized folds, and they become more obvious as receptor staining outside these regions becomes less common (Fig. 11, solid arrows).

We have noticed that in nearly all junctions the radial pattern mentioned above (see Fig. 6) also is associated with an emerging asymmetry between the two sides of the oval plaque. It was noted previously that the multiple axons entering a neuromuscular junction invariably entered from the same side (Balice-Gordon and Lichtman, 1993). When we restudied those images, we noted that the entry side of the nerve was associated with an irregular border of the receptor plaque (see Fig. 7) [see also Balice-Gordon and Lichtman (1993), their Figs. 2, 9, 10, 14-16]. One side (opposite the point of nerve entry and the origin of the radial branching pattern) maintains its oval shape, whereas the other side is more irregular. This asymmetry is increasingly prevalent in the second postnatal week. Even young adult junctions maintain this asymmetry in that the branches opposite the nerve entry zone usually trace out an oval pattern (Fig. 12). We have found that the irregular surface can be on either the left or right relative to the long axis of the muscle fiber or the upper or lower border. These various positions for the irregular surface are consistent with the fact that the nerve enters the junction from either side or from the upper or lower border.

By the end of the second postnatal week most of the junctions appear to have made the transformation from plaque to fully branched form (Fig. 13). Although the branches are crowded together, each appears as a well defined gutter depressed below the surrounding muscle fiber membrane.

In addition, by the end of the second postnatal week most superficial areas no longer show evidence of AChRs, suggesting that, albeit small, junctions have attained their mature receptor distribution.

\section{Denervation in the second postnatal week}

To determine, once gutter formation and AChR loss had begun, how autonomous these processes were, we denervated the sternomastoid muscle during the second postnatal week and then viewed the junctions either 3 d later (denervations on P07, $n=$ 19) or 1 week later at a time when junctions normally appear to be relatively mature (denervations on $\mathrm{P} 10, n=10$ ). We found that denervated junctions appeared not to progress at all from the time of their denervation. Furthermore, in the case of the longer denervations the junctions appeared to regress. Thus junctions denervated at P07 and viewed 3 d later retained characteristics seen at P07. For example, the denervated junctions showed rela- 


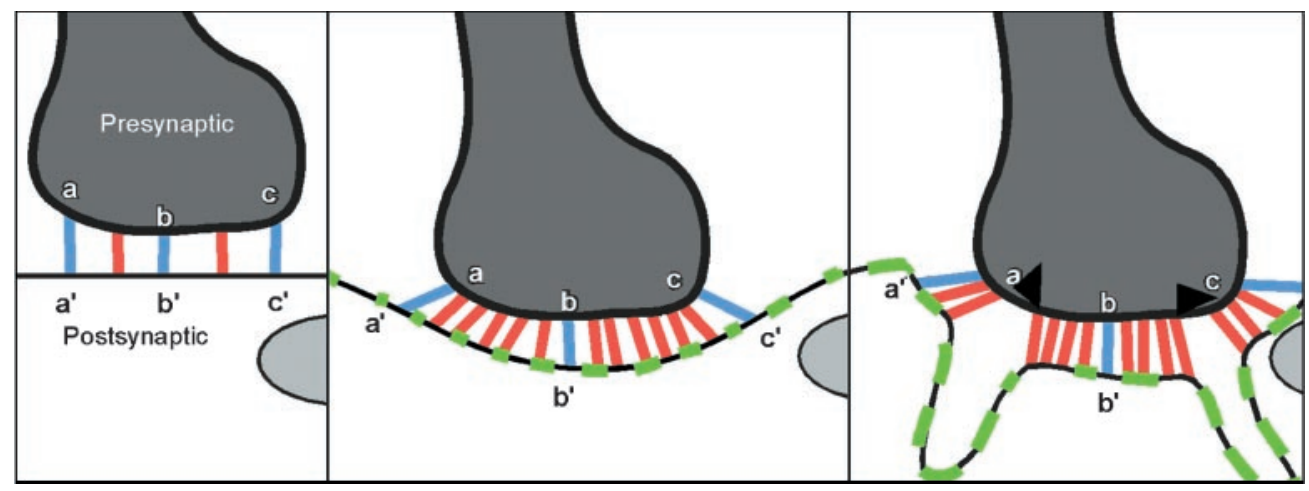
inserted in the membrane. This insertion causes an intercalary expansion of the postsynaptic site (Balice-Gordon and Lichtman, 1990;

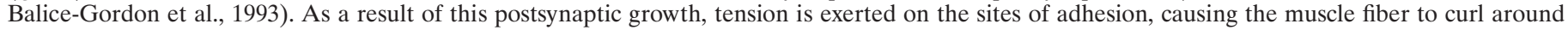

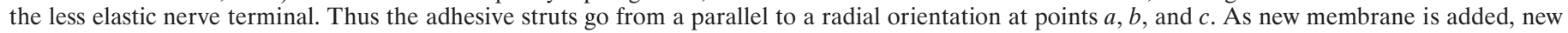

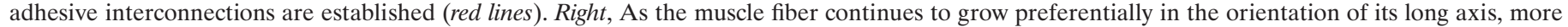

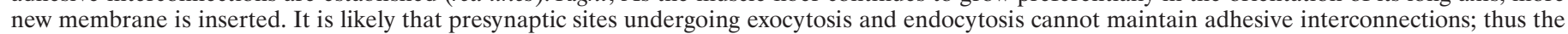
underlying postsynaptic membrane can infold at presynaptic active zones (black triangles).

tively few perforations as compared with contralateral control muscles and were often still oval-shaped. In addition, there were less obvious gutters. In junctions denervated at P10 and viewed at P17 we found evidence of gutter formation, and fold elaboration was only partially present (Fig. 14). Last, there were only hints of areas that were in the process of losing AChRs (Fig. 14).

\section{DISCUSSION}

This work adds to the extensive literature on neuromuscular junction structure by providing a topographical representation of location of AChRs. Additionally, this is the first optical technique capable of generating a high-resolution topography of the junctional folding pattern; it requires no sectioning or nerve removal procedure as is needed in electron microscopical approaches.

We found that during the first few postnatal weeks the postsynaptic membrane at individual neuromuscular junctions is a mosaic of two different regions. Some regions of the membrane are less reflective and have less organized folding, whereas other areas show folds and are associated with newly forming gutters. The transition from polyneuronal to single innervation is associated with a disappearance of AChRs at the underlying postsynaptic sites (Balice-Gordon and Lichtman, 1993; Gan and Lichtman, 1998). It is possible that the sites that showed reduced AChR reflection are associated with synapses that are eliminated. This suggests that eliminated synapses generally may not be associated with receptor-rich postsynaptic membranes having primary synaptic clefts and secondary folds. It is not clear, however, whether the absence of folds at sites losing AChRs is because such sites never differentiated folds or, alternatively, because these sites have undergone a process of dedifferentiation.

This work provides insight into the mechanism that generates the patterns of branches within a neuromuscular junction and the arrangement of folds within each branch. We saw that the nascent gutters of the future primary synaptic clefts fan out in a radial pattern in the oval plaque of AChRs (see, for example, Figs. 6, $10)$. The entry site also is associated with postsynaptic remodeling, because virtually all junctions became asymmetric; the outlines of the original oval AChR plaque remained evident at those edges of the junction that are not near the site of nerve entry, whereas the loss of AChRs disrupted the oval outline at the entry zone. Because the multiple inputs always approach the plaque from the same side (Balice-Gordon and Lichtman, 1993) and probably innervate the muscle shortly after entering the junction, the loss of all but one axon will lead inevitably to the disappearance of some nerve terminal sites near the entry zone. In addition, myelination of the preterminal axon can extend gradually into the entry zone of the neuromuscular junction. Myelination also causes a loss of AChRs at sites in which the overlying nerve becomes ensheathed [Balice-Gordon and Lichtman (1993), their Fig. 16]. Thus the nerves play a role in determining the branching pattern in the pretzel-shaped neuromuscular junction.

The muscle, however, is also apparently responsible for dictating some aspects of synaptic organization. We observed that mammalian junctions often have folds that run transverse to the long axis of the muscle fiber irrespective of the orientation of the gutters (see Fig. 1). Thus when junctional gutters were running in the long axis of the muscle fiber, the folds were transverse to the gutters, but when the gutters were running transverse to the long axis, the folds often were parallel to the gutter orientation. Previous scanning electron microscopical studies based on smaller sample sizes had noted that folds often were transverse to gutters (Ogata and Yamasaki, 1985; Desaki and Uehara, 1987), which may be related to the fact that more gutters run along the long axis of the muscle fiber than in the transverse direction (see Fig. 13). Similarly, in frog neuromuscular junctions the folds are also generally transverse to the long axis of the primary synaptic clefts, but these gutters also predominantly run parallel to the long axis of the fibers (Shotton et al., 1979). Thus, in contrast to the situation for the organization of the branches of the primary synaptic cleft, the pattern of junctional folds seems to be imposed at least partially by the orientation of the muscle fiber.

This ability of the muscle to determine the orientation of the junctional folds has motivated us to consider an explanation for the origin of junctional folds based on the forces exerted on the postsynaptic membrane as a consequence of the growth of muscle fibers and adhesion to the nerve. Analogous arguments have related tension forces that act in the developing brain to the origin of sulci and gyri and other structural features of the CNS (Van Essen, 1997). The hypothesis we came up with also provides a conceptual framework for thinking about the origin of three other topographical features observed in this study: the concavity of the junctional gutters, the concavity of the early spoon-shaped junctional plaque, and the reasoning for active zones that overlie postsynaptic folds. 
We consider the consequences for the topography of the synapse that result from acknowledging that sites of synaptic contact are tightly adhesive for the pre- and postsynaptic membranes. Evidence for this strong adhesion comes from the requirement of harsh acid or proteolytic treatments to strip nerve terminals from muscle fibers (Kuffler and Yoshikami, 1975; Fahim et al., 1983; Wilkinson and Lunin, 1994). We also consider the fact that the elongation and widening of muscle fibers during normal growth are associated with intercalary membrane addition throughout the muscle fiber, as seen in the intercalary enlargement of the AChR distribution as muscle fibers grow (Balice-Gordon and Lichtman, 1990; Balice-Gordon et al., 1990).

The intercalary membrane addition in muscle fibers thus may cause the established sites of adhesion with the nerve terminal via the intervening basal lamina to be pushed apart by new membrane insertion in the growing muscle fiber. If the connective tissue between the nerve and muscle is relatively inelastic, the adhesion between nerve terminals and muscle fibers will exert forces on the muscle fiber membrane associated with muscle growth. The direction of the tension forces will require either that the muscle fiber curl around the nerve and embrace it and/or that the nerve stretch to keep the adhesive sites in the basal lamina from breaking (Fig. 15). The concave shape of the AChR plaque in neonatal animals thus may be a consequence of the inability of the nerve to enlarge as fast as the expansion of the muscle fiber membrane. Disproportionate growth between muscle and nerve also may be the explanation for the subsequent sinking of the synaptic gutters as the muscle continues to add membrane at sites near contact with less quickly growing nerve terminal branches (Fig. 15).

If preferential muscle fiber growth is not matched by an equivalently rapid growth of the nerve terminal, then the nerve will prevent the elongation of the postsynaptic territory attached to it. In the presence of this restraining force any newly inserted muscle fiber membrane would have to fold to accommodate the constraints imposed by attachment to the nerve terminal. One location in which such infolding might occur is at sites in which there are few adhesive connections between the nerve and the underlying muscle. Adhesive connections are thought to occur around, but not at, release sites of central synapses (Uchida et al., 1996). Similarly, at the neuromuscular junction there is evidence of adhesive struts adjacent to active zones but an absence of adhesion at the release sites in which the basal lamina pulls away slightly from the nerve terminal [see, for example, Heuser (1980), his Fig. 7]. Muscle fiber membrane expansion thus would be selectively permissible at those sites, explaining why active zones overlie postsynaptic folds. In addition, if in early postnatal life muscle growth happens to be preferential in the long axis of the fiber, then the preferred orientation of folds would be transverse to the long axis.

This conceptual framework also provides a potential explanation for the loss of folds seen in denervated adult muscles although AChRs are maintained (Loring and Salpeter, 1980) and for the complete arrest in fold and gutter formation in development that follows the denervation described here. In adult denervated muscles the disappearance of folds may be accounted for by the membrane loss associated with muscle fiber atrophy. Consistent with this is that there is a 1-2 week lag before folds disappear and a similar lag before atrophy is severe. On the other hand, the absence of tension forces associated with nerve in neonatal denervation could be the reason that fold and cleft formation ceases after denervation in neonates.
The fact that the depths of folds vary between muscle types and species also may be explained by differences in the growth potential or elasticity of nerve terminals. For example, human neuromuscular junctions are typically smaller than mouse junctions, although often they are located on muscle fibers that are much larger. Human junctions, however, do have much longer junctional infoldings and deeper gutters (Engel and Santa, 1971). Thus the relative lack of nerve elongation in human muscle may explain why the folds and clefts are so deep and complex but the junctions are so small when compared with mouse. Although such mechanical explanations do not explain the functional significance of folds, they may be helpful in considering a number of questions related to the alignment of folds with active zones and the reasons why certain molecular perturbations cause changes in junctional folding (see, for example, Deconinck et al., 1997; Grady et al., 1997).

\section{REFERENCES}

Balice-Gordon RJ, Lichtman JW (1990) In vivo visualization of the growth of pre- and postsynaptic elements of mouse neuromuscular junctions. J Neurosci 10:894-908.

Balice-Gordon RJ, Lichtman JW (1993) In vivo observations of pre- and postsynaptic changes during the transition from multiple to single innervation at developing neuromuscular junctions. J Neurosci 13:834-855.

Balice-Gordon RJ, Breedlove SM, Bernstein S, Lichtman JW (1990) Neuromuscular junctions shrink and expand as muscle fiber size is manipulated: in vivo observations in the androgen-sensitive bulbocavernosus muscle of mice. J Neurosci 10:2660-2671.

Balice-Gordon RJ, Chua CK, Nelson CC, Lichtman JW (1993) Gradual loss of synaptic cartels precedes axon withdrawal at developing neuromuscular junctions. Neuron 11:801-815.

Bennett MR, Pettigrew AG (1974) The formation of synapses in striated muscle during development. J Physiol (Lond) 241:515-545.

Colman H, Nabekura J, Lichtman JW (1997) Alterations in synaptic strength preceding axon withdrawal. Science 275:356-361.

Deconinck AE, Potter AC, Tinsley JM, Wood SJ, Vater R, Young C, Metzinger L, Vincent A, Slater CR, Davies KE (1997) Postsynaptic abnormalities at the neuromuscular junctions of utrophin-deficient mice. J Cell Biol 136:883-894.

Desaki J, Uehara Y (1987) Formation and maturation of subneural apparatuses at neuromuscular junctions in postnatal rats: a scanning and transmission electron microscopical study. Dev Biol 119:390-401.

Engel AG, Santa T (1971) Histometric analysis of the ultrastructure of the neuromuscular junction in myasthenia gravis and in the myasthenic syndrome. Ann NY Acad Sci 183:46-63.

Fahim MA, Holley JA, Robbins N (1983) Scanning and light microscopic study of age changes at a neuromuscular junction in the mouse. J Neurocytol 12:13-25.

Flucher BE, Daniels MP (1989) Distribution of $\mathrm{Na}^{+}$channels and ankyrin in neuromuscular junctions is complementary to that of acetylcholine receptors and the $43 \mathrm{kDa}$ protein. Neuron 3:163-175.

Gan WB, Lichtman JW (1998) Synaptic segregation at the developing neuromuscular junction. Science 282:1508-1511.

Grady RM, Merlie JP, Sanes JR (1997) Subtle neuromuscular defects in utrophin-deficient mice. J Cell Biol 136:871-882.

Heuser J (1980) 3-D visualization of membrane and cytoplasmic specializations at the frog neuromuscular junction. In: Ontogenesis and functional mechanisms of peripheral synapses (Taxi J, ed), pp 139-155. France: INSERM Symposium No. 13.

Kelly AM, Zacks SI (1969) The fine structure of motor endplate morphogenesis. J Cell Biol 42:154-169.

Kuffler SW, Yoshikami D (1975) The distribution of acetylcholine sensitivity at the postsynaptic membrane of vertebrate skeletal twitch muscle: iontophoretic mapping in the micron range. J Physiol (Lond) 244:703-730.

Lichtman JW, Jhaveri S, Frank E (1984) Anatomical basis of specific connections between sensory axons and motor neurons in the brachial spinal cord of the bullfrog. J Neurosci 4:1754-1763.

Loring R, Salpeter MM (1980) Denervation increases turnover rate of junctional acetylcholine receptors. Proc Natl Acad Sci USA 77:2293-2298. 
Matthews-Bellinger JA, Salpeter MM (1983) Fine structural distribution of acetylcholine receptors at developing mouse neuromuscular junctions. J Neurosci 3:644-657.

Nyström B (1968) Postnatal development of motor nerve terminals in "slow-red" and "fast-white" cat muscles. Acta Neurol Scand 44:363-383.

Ogata T, Yamasaki Y (1985) The three-dimensional structure of motor endplates in different fiber types of rat intercostal muscle. A scanning electron microscopic study. Cell Tissue Res 241:465-472.

Padykula HA, Gautier GF (1970) The ultrastructure of the neuromuscular junctions of mammalian red, white, and intermediate skeletal muscle fibers. J Cell Biol 46:27-41.

Salpeter MM (1987) Vertebrate neuromuscular junctions: general morphology, molecular organization, and functional consequences. In: The vertebrate neuromuscular junction, pp 17-27. New York: Liss.

Sanes J, Lichtman JW (1999) Development of the vertebrate neuromuscular junction. Annu Rev Neurosci 22:389-442.
Shotton DM, Heuser JE, Reese BF, Reese TS (1979) Postsynaptic membrane folds of the frog neuromuscular junction visualized by scanning electron microscopy. Neuroscience 4:427-435.

Slater CR (1982) Postnatal maturation of nerve-muscle junctions in hindlimb muscles of the mouse. Dev Biol 94:11-22.

Steinbach JH (1981) Developmental changes in acetylcholine receptors at rat skeletal neuromuscular junctions. Dev Biol 84:267-276.

Teraväinen H (1968) Development of the myoneural junction in the rat. Z Zellforsch Mikrosk Anat 87:249-265.

Uchida N, Honjo Y, Johnson KR, Wheelock MJ, Takeichi M (1996) The catenin/cadherin adhesion system is localized in synaptic junctions bordering transmitter release zones. J Cell Biol 135:767-779.

Van Essen DC (1997) A tension-based theory of morphogenesis and compact wiring in the central nervous system. Nature 385:313-318.

Wilkinson RS, Lunin SD (1994) Properties of "reconstructed" motor synapses of the garter snake. J Neurosci 14:3319-3332. 